\title{
A randomised controlled trial to investigate ambulatory blood pressure response to riboflavin supplementation in adults with the MTHFR 677TT genotype
}

\author{
C.F. Hughes ${ }^{1}$, M. Ward ${ }^{1}$, A. McMahon ${ }^{1}$, J.J. Strain ${ }^{1}$, R. Plumb ${ }^{2}$, P. Weber ${ }^{3}$, \\ I. Bendik ${ }^{3}$ and H. McNulty ${ }^{1}$ \\ ${ }^{1}$ Nutrition Innovation Centre for Food and Health, Ulster University, Coleraine, BT52 1SA, ${ }^{2}$ School of Medicine, \\ Dentistry and Biomedical Science, Queens University Belfast, BT71NN and ${ }^{3}$ DSM Nutritional Products Ltd, \\ Kaiseraugst, Switzerland.
}

Hypertension is the leading cause of preventable premature death, estimated to affect over 1 billion adults worldwide. Meta-analyses of epidemiological studies suggest that the C677 T polymorphism in the gene MTHFR encoding the folate metabolising enzyme methylenetetrahydrofolate reductase (MTHFR) is associated with an increased risk of hypertension by $24-87 \%{ }^{(1)}$. Supplementation with riboflavin (a cofactor for MTHFR) can modify this phenotype as demonstrated in a series of randomised controlled trials conducted at this centre ${ }^{(1)}$. Compared with clinic blood pressure measured at one time-point only, ambulatory blood pressure monitoring (ABPM) measures BP over a $24 \mathrm{hr}$ period and provides a more reliable assessment of an individual's $\mathrm{BP}^{(2)}$. The aim of this study was to utilise ABPM to investigate the effect of riboflavin supplementation on BP in adults with the MTHFR 677TT genotype.

Adults 18-65yrs recruited across Northern Ireland to the RIBOGENE study (NCT02463513) were screened for the MTHFR TT genotype and those who were eligible and willing to participate $(n=81)$, were stratified by baseline systolic BP and randomised to receive either riboflavin treatment $(10 \mathrm{mg} /$ day) or placebo for 16 weeks. Biomarker status of riboflavin was measured using the erythrocyte glutathione reductase activation coefficient (EGRac) assay and BP was measured by both clinic BP and ABPM, in accordance with NICE guidelines ${ }^{(3)}$.

$\mathrm{BP}$ response to riboflavin was found to be strongly dependent on baseline BP. Participants with a baseline systolic BP of less than $125 \mathrm{mmHg}$ showed no response to riboflavin supplementation (data not shown). In participants with a baseline systolic BP $\geq 125 \mathrm{mmHg}$, riboflavin supplementation resulted in a significant BP lowering of daytime systolic $\mathrm{BP}$ by $3.8 \mathrm{mmHg}$ compared to $0 \cdot 2 \mathrm{mmHg}$ in the placebo group (see Table).

\begin{tabular}{|c|c|c|c|c|c|}
\hline & \multicolumn{2}{|c|}{ Riboflavin n23 } & \multicolumn{2}{|c|}{ Placebo n26 } & \multirow[b]{2}{*}{ P-value } \\
\hline & Mean & SD & Mean & SD & \\
\hline Age & $48 \cdot 6$ & $11 \cdot 3$ & $47 \cdot 8$ & $12 \cdot 0$ & $0 \cdot 823$ \\
\hline Male $n(\%)$ & 17 & $73 \cdot 9$ & 12 & $46 \cdot 2$ & 0.093 \\
\hline BMI & $28 \cdot 8$ & $3 \cdot 8$ & $27 \cdot 2$ & $3 \cdot 1$ & $0 \cdot 131$ \\
\hline Riboflavin status $^{\dagger}$ & Mean & $95 \% \mathrm{CI}$ & Mean & $95 \%$ CI & \\
\hline Pre & $1 \cdot 33$ & $1 \cdot 28-1 \cdot 38$ & 1.29 & $(1 \cdot 24-1 \cdot 34)$ & \\
\hline Post & $1 \cdot 20$ & $1 \cdot 14-1 \cdot 25$ & $1 \cdot 31$ & $(1 \cdot 25-1 \cdot 36)$ & $<0.001$ \\
\hline Change & \multicolumn{2}{|c|}{$-0 \cdot 12$} & \multicolumn{2}{|r|}{0.02} & \\
\hline Daytime BP $\mathrm{mmHg}^{\neq}$ & Mean & $95 \% \mathrm{CI}$ & Mean & $95 \%$ CI & \\
\hline Pre & $137 \cdot 10$ & $132 \cdot 7-141 \cdot 3$ & $134 \cdot 7$ & $130 \cdot 3-139 \cdot 1$ & \\
\hline Post & $133 \cdot 0$ & $128 \cdot 9-138 \cdot 1$ & $134 \cdot 8$ & $130 \cdot 6-139 \cdot 1$ & $0 \cdot 036$ \\
\hline Change & \multicolumn{2}{|c|}{$-3 \cdot 8$} & \multicolumn{2}{|r|}{$-0 \cdot 2$} & \\
\hline
\end{tabular}

Response to intervention analysed by repeated measures ANCOVA, adjusting for sex. ${ }^{\dagger}$ Higher EGRac values are indicative of lower riboflavin status. ${ }^{\neq}$mean of participant daytime/awake hrs which was personalised for each participant.

This is the first study to use ABPM to show that riboflavin supplementation, targeted at adults with the MTHFR 677TT genotype, results in significant lowering of mean day and night BP. It is also the first demonstration of this genotype specific effect of riboflavin on BP in non-hypertensive and younger adults. Given the frequency of this genotype worldwide (approximately 10\%, but as high as $30 \%$ in some populations) and the global burden of blood pressure-related disease, these findings could offer a personalised approach for BP management in these at risk sub-populations.

This work was funded by DSM Nutritional Products, Ltd. The support of the Wellcome Trust- Wolfston Northern Ireland Clinical Research Facility is acknowledged.

1. McNulty et al. (2017) Molecular Aspects of Medicine 53, $2-9$.

2. McMahon et al. (2016) Nutrients 8, 720. doi:10.3390/nu8110720

3. National Institute for Health and Care Excellence (2011) Hypertension in adults: diagnosis and management nice.org.uk/guidance/cg127. 